\title{
Séminaires / Seminari
}

Bien qu'environ $40 \%$ de tous les médecins ouvrent tôt ou tard leur propre cabinet médical et ont besoin de connaissances en gestion d'entreprise hormis leur savoir-faire médical, cette thématique n'est souvent pas ou peu abordée. FMH Services qui s'occupe de la gestion d'entreprise des membres FMH comble désormais cette lacune en proposant des séminaires spécifiques. Dans ces séminaires, les médecins peuvent acquérir les bases nécessaires pour ouvrir leur propre cabinet médical ou un cabinet de groupe ou pour remettre leur cabinet de manière optimale. Les médecins obtiennent également des conseils sur la manière de collaborer avec les autorités ou avec des spécialistes tels que des experts-comptables, des conseillers financiers, en assurance ou en crédits bancaires, des juristes, des architectes, etc. Ces différents thèmes sont abordés par des experts qualifiés de la FMH et de FMH Services.

\section{Ouverture et gestion d'un cabinet médical}

\section{Participants}

Le séminaire est destiné aux médecins sur le point d'ouvrir ou de reprendre un cabinet médical.

\section{Contenu}

La réalisation d'un cabinet médical ne s'improvise pas. Vous allez dépendre du financement et de la gestion de votre cabinet médical. Ce séminaire traitera de l'environnement socio-politique qui sera le vôtre en tant qu'indépendant, et vous permettra de vous y préparer. Les thèmes suivants seront développés lors de ce séminaire: l'aménagement, le financement, les assurances, la fiscalité, la comptabilité et l'administration de votre cabinet.

\section{Documentation}

Basé sur une check-liste pour l'ouverture d'un cabinet médical, le classeur remis à chaque participant contient toutes les informations importantes.

\section{Frais / Sponsors}

Le séminaire est gratuit pour les membres FMH. Les frais sont couverts par les sponsors suivants: Caisse-Maladie des Médecins Suisses, Axon Lab SA et AstraZeneca SA
Date

No 19 Jeudi 18 septembre 2003 Hostellerie Genève $\quad 9.00-17.00 \mathrm{~h}$ de la Vendée

\section{Inscription}

Vous pouvez vous inscrire à l'adresse mentionnée ci-dessous ou via internet: www.fmhservices.ch.

\section{Remise/reprise d'un cabinet médical}

\section{Participants}

Le séminaire s'adresse aux médecins désirant remettre ou reprendre un cabinet médical.

\section{Contenu}

Questions principalement abordées: explication des aspects juridiques de la remise et de la reprise d'un cabinet médical (contrat de remise de cabinet, transfert des dossiers médicaux, etc.), mesures architecturales à envisager et coût de cellesci, questions d'assurance à mettre au point de part et d'autre (par exemple planification de la prévoyance, reprise de contrats d'assurance), estimation de la valeur du cabinet (valeur d'inventaire et goodwill) et conséquences fiscales d'une remise ou d'une reprise de cabinet (moment idéal de remise, impôt sur la liquidation et le bénéfice de l'immeuble, etc.).

\section{Documentation}

Les participants du séminaire reçoivent un classeur et les documents contenant des conseils pratiques au sujet de la remise ou de la reprise d'un cabinet médical.

\section{Frais / Sponsor}

Le séminaire est gratuit pour les membres FMH. Les frais sont couverts par le sponsor suivant: CaisseMaladie des Médecins Suisses et AstraZeneca SA

\section{Date}

$\begin{array}{lll}\text { No } 21 \text { Jeudi } 6 \text { novembre } 2003 & \text { Hostellerie } \\ \text { Genève } 9.00-16.30 \mathrm{~h} & \text { de la Vendée }\end{array}$

\section{Inscription}

Vous pouvez vous inscrire à l'adresse mentionnée ci-dessous ou via internet: www.fmhservices.ch. 


\section{Cours d'introduction à I'ordinateur}

\section{Participants}

Le cours s'adresse aux médecins sur le point d'ouvrir un cabinet médical ou aux médecins déjà établis.

\section{Contenu}

L'exposé d'introduction informe les participants sur les applications informatiques disponibles pour le cabinet médical. Les participants peuvent ensuite se familiariser avec des logiciels concernant la gestion du cabinet médical, proposés par quatre entreprises différentes qui leur proposent un aperçu des programmes les plus performants. Ils seront ainsi à même de choisir la solution informatique la plus appropriée et de savoir quelles sont les erreurs à éviter.

\section{Frais}

Le cours est gratuit pour les membres FMH.

\section{Date}

No 23 Jeudi 25 septembre 2003 Beaulieu Lausanne $14.00-17.30 \mathrm{~h}$ Exploitation SA

\section{Les entreprises suivants vous pouvez connaître:}

Caisse des Médecins, Thônex (Mediwin CB Pro) 40 ans d'existence, et 14 ans de facturation par PC, la Caisse des Médecins a su profiter de l'expérience acquise pour développer des produits adaptés aux besoins réels du cabinet médical. Ses logiciels dont la dernière version a été particulièrement développée pour le TARMED ont depuis longtemps intégré la facturation électronique des données et obtenu le label «programme gestion confiance» délivré par la commission informatique de l'AMG. L'implantation régionale est, en plus, un atout supplémentaire pour le cabinet médical.
BB-Soft, Lausanne (MédiACT)

Créé en 1988 et dirigé par un médecin non praticien, BB-Soft a conçu et réalisé MédiACT avec une idée directrice: votre confort. Sur Macintosh ou sur PC (Windows), grande facilité d'usage et rapidité d'apprentissage. Agréé comme programme de confiance par l'Asscociation des Médecins de Genève.

GNT, Gespower New Technologies, Chêne-Bourg (MEDISOFT 5 Tarmed)

GNT développe des applications dans le domaine médical, médecins et laboratoires d'analyses. Nos produits sont commercialisés à l'échelle européenne. Médisoft 5 Tarmed est un logiciel complet, puissant et convivial. Il répond totalement aux besoins d'un cabinet médical de 1 à 50 médecins. Modernité et richesse de fonctionnalités à faible coût. Médisoft 5 Tarmed est un programme de confiance de la commission informatique de l'AMG.

\section{PSIPI SA, Plan-les-Ouates / GE (PsiMed)}

PSIPI SA est spécialisée dans le développement de logiciels médicaux: gestion de cabinet médical, dossier médical électronique, imagerie, connexions aux appareils médicaux, échanges électroniques entre médecins, laboratoires, hôpitaux, bases de données externes. Extrême souplesse de paramétrisation des applications et facilité d'utilisation. Synergie entre les produits et les services PSIPI SA et d'autres acteurs du domaine de la santé: médicaments, assurances, communications sécurisées. PsiMed est agréé programme de confiance de l'Association des Médecins de Genève.

\section{Inscription}

Vous pouvez vous inscrire à l'adresse mentionnée ci-dessous ou via internet: www.fmhservices.ch. 


\section{Contabilità e Fiscalità (Ticino)}

\section{Contenuto}

L'obiettivo di questo seminario è di dare al medico le conoscenze necessarie per utilizzare autonomamente lo strumento contabile e per operare con più efficienza ed economicità, nonché far comprendere al medico il significato della pianificazione fiscale.

Mediante esempi pratici elaborati in gruppo, verrà illustrato in maniera semplice ma completa il sistema di gestione della contabilità di uno studio medico con effetto anche sulla pianificazione fiscale.

\section{Spese}

\begin{tabular}{ll} 
Membri ordinari o straordinari FMH & fr. $150 .-$ \\
\hline Per accompagnatori & fr. $75 .-$
\end{tabular}

\section{Date}

No 25 Giovedì 13 novembre 2003 FMH Services Chiasso dalle 9.00 alle 16.00 Fiduciaria

\section{Iscrizione}

Potete iscrivervi all'indirizzo sottindicato oppure via internet sotto il sito www.fmhservices.ch

\section{Inscription et information}

FMH Consulting Services, Mme Simone Köpfli, Burghöhe 1, 6208 Oberkirch, Tél. 04192500 77, Fax 0419210586 ou via internet: www.fmhservices.ch.

\section{Conditions d'annulation}

Pour les séminaires gratuits (à l'exclusion des séminaires sur informatique au cabinet médical), un montant est perçu pour absence ou annulation. Il est de:

- Fr. 50.- par personne dans les 15 jours avant et

- Fr. 80.- par personne dans les 7 jours avant le début du séminaire. 